\title{
The prognostic significance of left atrial appendage peak flow velocity in the recurrence of persistent atrial fibrillation following first radiofrequency catheter ablation
}

\author{
Wentao Yang ${ }^{1,2}$, Qing Zhao ${ }^{2}$, Minghui Yao ${ }^{3}$, Xiangdong Li $^{2}$, Yue Zhang ${ }^{2}$, Chuanbin Liu ${ }^{2}$, Zhaoliang Shan ${ }^{2}$, \\ Yutang Wang ${ }^{1,4}$ \\ ${ }^{1}$ College of Medicine, Nankai University, Tianjin, China; ${ }^{2}$ Department of Cardiology, The Sixth Medical Center, Chinese PLA General Hospital, \\ Beijing, China; ${ }^{3}$ Department of Cardiovascular Surgery, the Sixth Medical Center of PLA General Hospital, Beijing, China; ${ }^{4}$ Department of \\ Geriatric Cardiology, The Second Medical Center \& National Clinical Research Center for Geriatric Diseases, Chinese PLA General Hospital, \\ Beijing, China \\ Contributions: (I) Conception and design: Y Wang, W Yang; (II) Administrative support: Y Wang; (III) Provision of study materials or patients: None; \\ (IV) Collection and assembly of data: W Yang, Q Zhao, M Yao, X Li; (V) Data analysis and interpretation: W Yang, Q Zhao, Y Zhang, C Liu, Z \\ Shan; (VI) Manuscript writing: All authors; (VII) Final approval of manuscript: All authors. \\ Correspondence to: Yutang Wang. College of Medicine, Nankai University, 94 Weijin Rd., Tianjin 300071, China; Department of Geriatric Cardiology, \\ The Second Medical Center \& National Clinical Research Center for Geriatric Diseases, Chinese PLA General Hospital, 28 Fuxing Rd., Beijing \\ 100853, China. Email: wangyutang301@126.com.
}

Background: Atrial fibrillation (AF) relapse following radiofrequency catheter ablation (RFCA) for persistent atrial fibrillation (PeAF) continues to be a concern. This study establishes a connection between left atrial appendage peak flow velocity (LAAV) and recurrence of AF in individuals having PeAF following first RFCA.

Methods: We retrospectively studied 164 successive PeAF patients who had first RFCA between January 2018 and December 2019. Before the ablation, the LAAV was recorded using transesophageal echocardiography (TEE). The demographic and clinical data of the individuals were gathered. Participants were monitored at regular intervals to monitor for recurrence of AF. We employed Cox proportional hazards regression to determine if $\mathrm{LAAV}$, as well as other clinical indicators, were predictive of $\mathrm{AF}$ recurrence in follow-up.

Results: In this study, AF relapse was seen in 43 patients (26.2\%) following a median follow-up of 15 [interquartile range (IQR): 12-18] months. It was shown that the LAAV was decreased in individuals who had recurrences of $\mathrm{AF}(0.36 \pm 0.05$ vs. $0.45 \pm 0.17 \mathrm{~m} / \mathrm{s}, \mathrm{P}=0.004)$. Using Kaplan-Meier analysis, it was discovered that the low LAAV $(0.37 \mathrm{~m} / \mathrm{s})$ group had a poorer event-free survival rate compared to the high LAAV $(>0.37 \mathrm{~m} / \mathrm{s})$ group (17.6 vs. 21.2 months, log-rank $\mathrm{P}=0.002)$ group. Based on the results of the multivariate Cox regression analysis, a LAAV of fewer than $0.37 \mathrm{~m} / \mathrm{s}$ [hazard ratio (HR): 2.32; $95 \%$ confidence interval (CI): 1.177-4.227; $\mathrm{P}=0.014]$ was shown to be an independent predictor of AF recurrence following RFCA.

Conclusions: A low LAAV is associated with AF relapse, and it is a predictor of AF relapse following the first RFCA for PeAF. This discovery may be useful in the optimization of treatment strategies and the care of patients with PeAF.

Keywords: Left atrial appendage peak flow velocity (LAAV); persistent atrial fibrillation (PeAF); transesophageal echocardiography (TEE); radiofrequency catheter ablation (RFCA); recurrence

Submitted Jul 30, 2021. Accepted for publication Sep 27, 2021.

doi: $10.21037 /$ jtd-21-1363

View this article at: https://dx.doi.org/10.21037/jtd-21-1363

(C) Journal of Thoracic Disease. All rights reserved. 


\section{Introduction}

Clinically, atrial fibrillation (AF) is a usual heart arrhythmia correlated with an elevated risk of adverse effects like thromboembolic strokes, heart failure (HF), systemic embolism (SE), and mortality $(1,2)$. The incidence and burden of $\mathrm{AF}$ have risen dramatically in recent decades in China due to the aging population and continued vulnerability to risk factors (3). Radiofrequency catheter ablation (RFCA) is a successful method for treating AF. Although the advancement in ablation techniques has dramatically improved outcomes (4), post-ablation recurrence remains challenging. RFCA has proven to be promising for paroxysmal AF; however, the ideal ablation strategy for persistent atrial fibrillation (PeAF) is elusive. Using RFCA in PeAF often occurs AF recurrence, and the therapeutic benefit is limited even following multiple procedures (5-7). Consequently, finding reliable predictors of $\mathrm{AF}$ recurrence before ablation may help refine treatment strategies and manage patients with PeAF.

Certain variables, such as AF duration (8), body mass index (BMI) (9), high blood pressure (10), diabetes mellitus (11), left atrial (LA) diameter $(12,13)$, LA strain $(14)$, and other echocardiographic indexes $(15,16)$ can play a role in predicting ablation outcomes. However, no valid parameter exists to forecast $\mathrm{AF}$ recurrence following ablation. There is a lack of research about the left atrial appendage (LAA) in this situation. Previous research on patients with PeAF has shown that the LAA is critical in initiating and sustaining $\operatorname{AF}(5,17,18)$. Electrical isolation of the LAA enhanced independence from all-atrial arrhythmia relapse in non-paroxysmal AF individuals than the standard ablation only (17). Left atrial appendage peak flow velocity (LAAV) represents a hemodynamic feature of LA and the LAA (19-21). In addition, LAAV in PeAF patients showed an inverse relationship with the degree of LA lowvoltage areas (LVA) (22) that is considered a surrogate for LA fibrosis (23) and an important predictor of recurrent AF following catheter ablation $(24,25)$. Nevertheless, the LAAV role in predicting AF recurrence has not yet been established. A decreased LAAV enhances the likelihood of recurrence of PeAF following ablation in the 12 months follow-up time following the procedure (26-29). On the other hand, other research suggests that LAAV is not a reliable predictor of recurrence following catheter ablation of PeAF in all cases (30).

Given the above contradictory conclusions, this study aimed to investigate whether LAAV is an indicator of
AF recurrence in individuals with PeAF. We present the following article in accordance with the STARD reporting checklist (available at https://dx.doi.org/10.21037/jtd-211363).

\section{Methods}

\section{Study population}

We retrospectively examined the data for 164 consecutive PeAF patients who underwent a first RFCA at the Chinese PLA General Hospital between January 2018 and December 2019. PeAF was defined as an AF episode that lasted more than a week and necessitated medication administration or direct current cardioversion to terminate it. A history of cardiac surgery, moderate to severe mitral regurgitation or stenosis, chronic heart failure (CHF) [New York Heart Association (NYHA) $\geq$ III class], atrial or ventricular thrombus, current inflammation, hepatopathy, or hyperthyroidism were all excluded from consideration. In addition, all individuals were classified into two groups: those who maintained a stable sinus rhythm throughout follow-up (the "no recurrence group") and those who had an AF recurrence (recurrence group). All procedures performed in this study involving human participants were in accordance with the Declaration of Helsinki (as revised in 2013). The study was approved by the Human Ethics Review Committee of the Chinese PLA General Hospital and informed consent was taken from all the patients.

\section{Clinical data and measurement}

All participants' demographic and clinical data were collected, including age, origin, $\mathrm{BMI}$, medication usage, $\mathrm{AF}$ duration, prior history, and the medications.

In non-rheumatic AF individuals, the HAS-BLED and CHA2DS2-VASc ratings are used to predict the risk of bleeding or stroke. At our hospital's central lab, patients' blood specimens were examined for the uric acid (UA) levels and N-terminal pro-brain natriuretic peptide (NTproBNP) while they were in the hospital for treatment.

\section{Transesophageal echocardiography (TEE)}

TEE was conducted utilizing an IE Elite ultrasound furnished having multiplane TEE observation within 24 hours prior to RFCA. It was decided to look for the LA spontaneous echo contrast and thrombus. The LAA pictures 


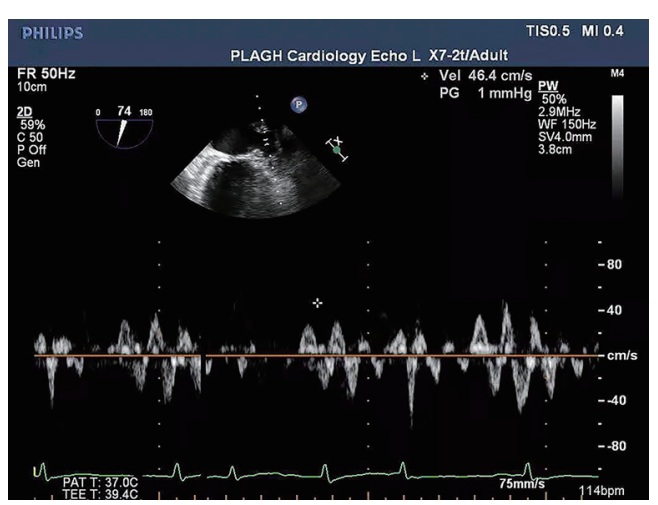

Figure 1 Example of LAAV measurement. LAAV, left atrial appendage peak flow velocity; TIS, soft tissue thermal index; MI, mechanical index; PLAGH, PLA General Hospital; FR, frame rate; 2D, two-dimensional; C, contrast; $\mathrm{P}$, persistence; Vel, velocity; PG, pressure gradient; PW, pulse wave; WF, wall filter; SV, sample volume; PAT, patient; TEE, transesophageal echocardiography; T, temperature.

were acquired using a transverse scan in the basal short-axis view and a vertical scan in the left ventricle-LA 2-chamber, using the left ventricle-LA 2-chamber observation being the most common (23). In order to estimate the peak flow velocity at the LAA entry (Figure 1), we utilized pulsedwave Doppler imaging, which was computed as the mean score of ten successive fibrillatory emptying waves. All TEE pictures were stored for further viewing offline.

Before the TEE analysis, 2D transthoracic echocardiography was conducted with the IE Elite and a 3.5 $\mathrm{MHz}$ transducer. Common observations and variables were registered from the apical and parasternal views. The left atrial diameter (LAD), left ventricle diameter (LVD), and left ventricle ejection fraction (LVEF) were measured.

\section{Electrophysiological study}

Patients received multidetector computed tomography (MDCT) scans of the LA and pulmonary veins (PVs) to guide the ablation process throughout the procedure. It was decided to utilise the CARTO 3 system (Biosense Webster, USA) to guide the circumferential pulmonary vein isolation (CPVI) procedure (31).

The following are the critical stages in the process: Initially, a single transseptal puncture was performed to investigate the LA, with the administration of unfractionated heparin (100 IU/kg). Using the CARTO 3 mapping system, the three-dimensional (3D) structure of the LA and PV trunks was reassembled using a PentaRay catheter (Biosense Webster, USA) in conjunction with the MDCT scan. Each PV antrum was subjected to a point-by-point irrigated radiofrequency ablation. At a temperature below $43{ }^{\circ} \mathrm{C}$, radiofrequency energy was used at $30-35 \mathrm{~W}$ and a $17 \mathrm{~mL} / \mathrm{min}$ flow rate for 30-35 seconds per point. Electrical separation of the PVs was the ablation termination point. Successful PV extraction was described as removing PV prospects obtained from a PentaRay catheter throughout the procedure. According to the electrophysiological mapping findings, ablation of the LA roofline and non-PV foci causing AF was performed as necessary. The electrophysiological analysis verified the block lines. Biphasic direct current shocks of $100-150 \mathrm{~J}$ were used to preserve the sinus beat if the AF continued after the ablation. Heparin was used once more in the process. The clotting time of full blood in activated form was calculated every 30 minutes to adjust the heparin dosage. During the procedure, intravenous fentanyl was used to maintain analgesia.

\section{AF recurrence assessment and follow-up}

Amiodarone was prescribed orally for 3 months postablation to patients with no contraindications. Patients were advised to stop using amiodarone and all other anti-arrhythmic drugs (AADs) if there was no atrial tachyarrhythmia. Vitamin K antagonists, rivaroxaban or dabigatran recommended for at least 3 months for patients without contraindications. Many of the patients received follow-up appointments for daily psychiatric evaluations. They were followed up with outpatient hospital visits every 3 months for the first year, and then every 6 months. Examinations, such as 12-lead electrocardiograms (ECGs) and 24-hour Holter recordings, were scheduled for patients between 3-24 months following the method. The total follow-up period was 24 months. When patients experienced symptoms after ablation, such as fatigue, palpitations, vertigo, or nausea, additional 24-hour Holter monitoring and ambulatory electrocardiograms were conducted. Phone interviews were scheduled, and patients were asked to send us their ECG via WeChat in the event of canceling their outpatient follow-up. After 3-month blanking period, the relapse of AF after ablation was described as $\mathrm{AF} /$ atrial flutter/atrial tachyarrhythmia lasting at least 30 seconds and registered by an ECG or device recording system (6). The clinical follow-up and data were analyzed separately by two electrophysiologists. 


\section{Statistical analysis}

To ensure data normal distribution, the KolmogorovSmirnov test $(\mathrm{P}>0.05)$ was performed. Continuous variables are represented as the mean \pm standard deviations (SDs) if they are regularly distributed, or as the median and quartile (Q1-Q3) if they are not normally distributed. Categorical variables are variables that have values associated with them (\%). We utilized the independent samples $t$-test and MannWhitney $\mathrm{U}$ test for continuous variables, and the Chisquare/Fisher Exact test for categorical variables to interpret the results. The cutoff value for LAAV in predicting AF recurrence following catheter ablation was determined via the use of a receiver operating characteristic (ROC) curve analysis. The Kaplan-Meier curve was used to calculate the cumulative AF recurrence rate after ablation. Age, sex, BMI, AF duration, LAD, LVD, LVEF, hypertension, Diabetes mellitus, CHF, coronary heart disease (CHD), NT-proBNP, and LAAV were entered into a univariate Cox proportional hazards regression model to recognize the parameters related to AF recurrence after RFCA. Variables with a $\mathrm{P}$ value $<0.20$ in the univariate analysis were then incorporated into a multivariate analysis to recognize the independent predictors of AF recurrence. The Spearman test was used to measure correlations between variables. We employed SPSS version 25 for all statistical analyses. All statistical analysis were two-sided, with a $\mathrm{P}$ value of 0.05 .

\section{Results}

\section{Baseline features}

Data from 164 PeAF patients (126 males and 38 females, mean age $58.2 \pm 9.7$ years) who had undergone their initial ablation were analyzed. According to their medical records, $43(26.2 \%)$ patients suffered AF recurrence, and 121 (73.8\%) patients had a steady sinus beat during the followup observation period \{median time 15 [interquartile range (IQR): 12-18] months\}. Participants were classified into two groups, dependent on AF recurred or not.

Baseline characteristics of all patients are summarized in Table 1.

The results showed that the recurrence group had a longer duration of $\mathrm{AF}$ and a higher NT-proBNP level $(\mathrm{P}<0.05)$. Viewing echocardiographic data, the LAD was substantially higher in the recurrence group, but the LAAV was significantly lower $(0.36 \pm 0.15$ vs. $0.45 \pm 0.17 \mathrm{~m} / \mathrm{s}, \mathrm{P}=0.004)$. There were no additional statistically significant differences among the 2 groups in terms of baseline characteristics.

\section{Baseline LAAV predictive value}

The baseline LAAV cut-off value to predict $\mathrm{AF}$ reappearance was $0.37 \mathrm{~m} / \mathrm{s}$, as determined by the ROC curve analysis (sensitivity: $60.5 \%$, specificity: $66.9 \%$ ). Figure 2 shows the area under the curve (AUC) value of $0.65[95 \%$ confidence interval (CI): 0.552-0.747, $\mathrm{P}=0.004]$. Based on LAAV cut-off value $(0.37 \mathrm{~m} / \mathrm{s})$, the 164 patients with PeAF were classified into two subgroups. The low LAAV group had a significantly shorter duration of event-free survival compared to the increased LAAV group (17.6 vs. 21.2 months, log-rank $\mathrm{P}=0.002$, Figure 3).

\section{Multivariate and univariate analyses of $A F$ recurrence following RFCA}

Table 2 summarizes the results of the multivariate and univariate analyses for $\mathrm{AF}$ recurrence.

The univariate analysis showed that AF relapse was significantly related to $\mathrm{AF}$ duration, $\mathrm{CHF}, \mathrm{LAD}$, and a LAAV $\leq 0.37 \mathrm{~m} / \mathrm{s}$. In the multivariate Cox regression, forward stepwise analysis adjusted for $\mathrm{AF}$ duration, $\mathrm{CHF}$, LAD, LAAV $\leq 0.37 \mathrm{~m} / \mathrm{s}$, CHD, NT-proBNP, and LVEF indicated that LAAV $\leq 0.37 \mathrm{~m} / \mathrm{s}$ [hazard ratio (HR): 2.32 , 95\% CI: 1.177-4.227, P=0.014], AF duration (HR: 1.007, 95\% CI: 1.002-1.013, $\mathrm{P}=0.006$ ), and LAD (HR: 1.124, 95\% CI: 1.011-1.250, $\mathrm{P}=0.032$ ) were independent predictors of $\mathrm{AF}$ relapse following RFCA.

\section{Correlations between LAAV and other variables}

For the echocardiographic data and lab findings and, Spearman rank correlation analysis demonstrated that LAAV was negatively associated with age $(r=-0.198$, $\mathrm{P}=0.011)$, CHA2DS2-VASc score $(\mathrm{r}=-0.25, \mathrm{P}=0.001)$, HASBLED score $(r=-0.185, P=0.017)$, NT-proBNP $(r=-0.125$, $\mathrm{P}=0.006)$, and $\operatorname{LAD}(\mathrm{r}=-0.125, \mathrm{P}=0.004)$. LAAV was positively associated with $\operatorname{LVEF}(\mathrm{r}=0.21, \mathrm{P}=0.007)$ (Table 3). We do not find any significant association between LAAV and any other parameters $(\mathrm{P}>0.05)$.

\section{Discussion}

This research examined the connection between preprocedural LAAV and AF recurrence in 164 PeAF patients who underwent initial RFCA. Our findings revealed that 26.2\% ( $\mathrm{n}=43)$ of PeAF patients suffered AF recurrence during the median follow-up period of 15 (IQR: 12-18) 
Table 1 Baseline characteristics of patients with and without $\mathrm{AF}$ recurrence

\begin{tabular}{|c|c|c|c|c|}
\hline Characteristics & Total $(n=164)$ & Recurrence $(n=43)$ & No recurrence $(n=121)$ & $P$ value \\
\hline Age (years) & $58.21 \pm 9.7$ & $58.51 \pm 9.03$ & $58.08 \pm 10.07$ & 0.844 \\
\hline $\mathrm{BMI}\left(\mathrm{kg} / \mathrm{m}^{2}\right)$ & $26.77 \pm 3.43$ & $26.72 \pm 3.81$ & $26.81 \pm 3.31$ & 0.957 \\
\hline Smoking, $\mathrm{n}(\%)$ & $68(41.5)$ & $16(37.2)$ & $52(43.0)$ & 0.509 \\
\hline Hypertension, n (\%) & $82(50.0)$ & $19(44.2)$ & $63(52.1)$ & 0.374 \\
\hline Diabetes mellitus, $\mathrm{n}(\%)$ & $25(15.2)$ & $8(18.6)$ & $17(14.0)$ & 0.475 \\
\hline Hyperlipemia, n (\%) & $44(26.8)$ & $13(30.2)$ & $31(25.6)$ & 0.502 \\
\hline CHD, n (\%) & $30(18.3)$ & $10(23.3)$ & $20(16.5)$ & 0.294 \\
\hline Peripheral arterial disease, $\mathrm{n}(\%)$ & $11(6.7)$ & $5(11.6)$ & $6(5.0)$ & 0.252 \\
\hline CHA2DS2-VASc score & $2[1-3]$ & $2[1-3]$ & $2[1-4]$ & 0.237 \\
\hline HAS-BLED score & $1[0-2]$ & $1[0-2]$ & $1[0-1.5]$ & 0.45 \\
\hline NT-proBNP (pg/mL) & $380[245-567]$ & 494 [346-643] & $342[232-521]$ & $<0.001^{*}$ \\
\hline $\mathrm{UA}(\mu \mathrm{mol} / \mathrm{L})$ & 357 [288-427] & $373[321-440]$ & $348[288-424]$ & 0.135 \\
\hline \multicolumn{5}{|l|}{ Echocardiographic data } \\
\hline LAD (mm) & $41[38-45]$ & $43[39-46]$ & $41[38-43]$ & $0.013^{*}$ \\
\hline LVD (mm) & 46 [44-50] & $47[44-50]$ & 46 [44-50] & 0.583 \\
\hline Propafenonen & $32(19.5)$ & $10(23.3)$ & $22(18.2)$ & 0.470 \\
\hline$\beta$-blockers & $74(45.1)$ & $22(51.2)$ & $52(43.0)$ & 0.354 \\
\hline ACEI/ARB & $48(29.3)$ & $15(34.8)$ & $33(27.3)$ & 0.346 \\
\hline Statin & $102(62.2)$ & $24(55.8)$ & $78(64.5)$ & 0.315 \\
\hline \multicolumn{5}{|l|}{ Ablation procedures, n (\%) } \\
\hline Additional roof ablation & $42(25.6)$ & $13(30.2)$ & $29(24.0)$ & 0.418 \\
\hline
\end{tabular}

*, $\mathrm{P}<0.05$. AF, atrial fibrillation; BMI, body mass index; CHD, coronary heart disease; CHF, chronic heart failure; CHA2DS2-VASc, Congestive Heart Failure, Hypertension, Age $\geq 75$ years [doubled], Diabetes Mellitus, Stroke [doubled], Vascular Disease, Age (65-74 years), Sex category [female]; HAS-BLED, elderly, history of hypertension, history of abnormal renal or liver function, history of stroke, history of major bleeding, medication use predisposing to bleeding, alcohol use, Labile international normalized ratio; NT-proBNP, $\mathrm{N}$-terminal pro-brain natriuretic peptide; UA, uric acid; LAD, left atrial diameter; LVD, left ventricular diameter; LVEF, left ventricular ejection fraction; LAAV, left atrial appendage peak flow velocity; NOAC, new oral anticoagulants; ACEI, angiotensin-converting enzyme inhibitors; $A R B$, angiotensin receptor blocker. 


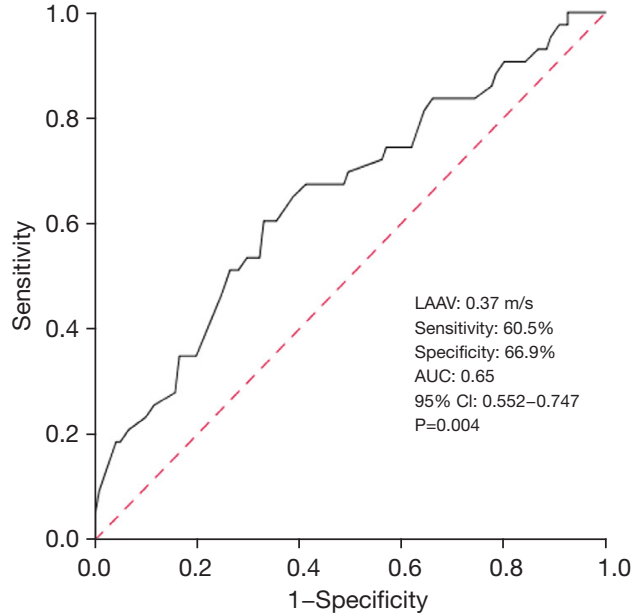

Figure 2 ROC curve analysis evaluating the LAAV value in predicting AF recurrence. ROC, receiver operating characteristic; LAAV, left atrial appendage peak flow velocity; AF, atrial fibrillation; AUC, area under the curve; CI, confidence interval.

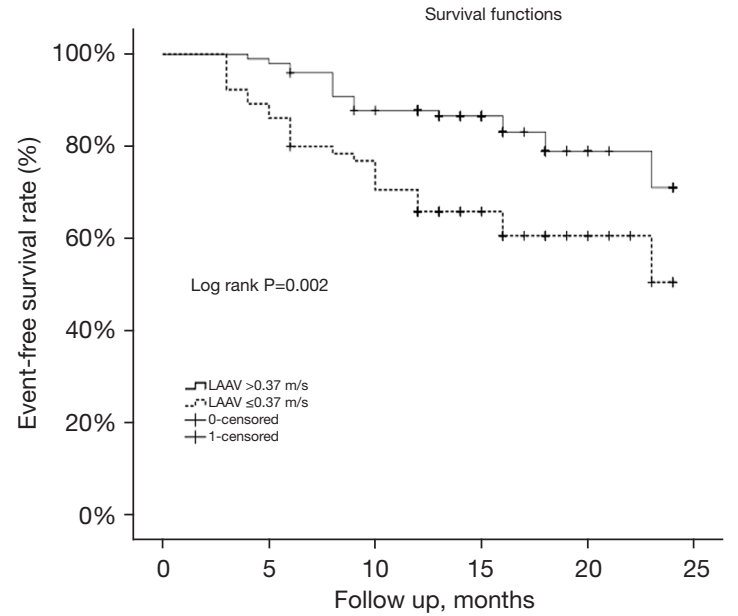

Figure 3 Kaplan-Meier curves of event-free survival rate by LAAV. LAAV, left atrial appendage peak flow velocity.

Table 2 Univariate and multivariate analyses of AF recurrence

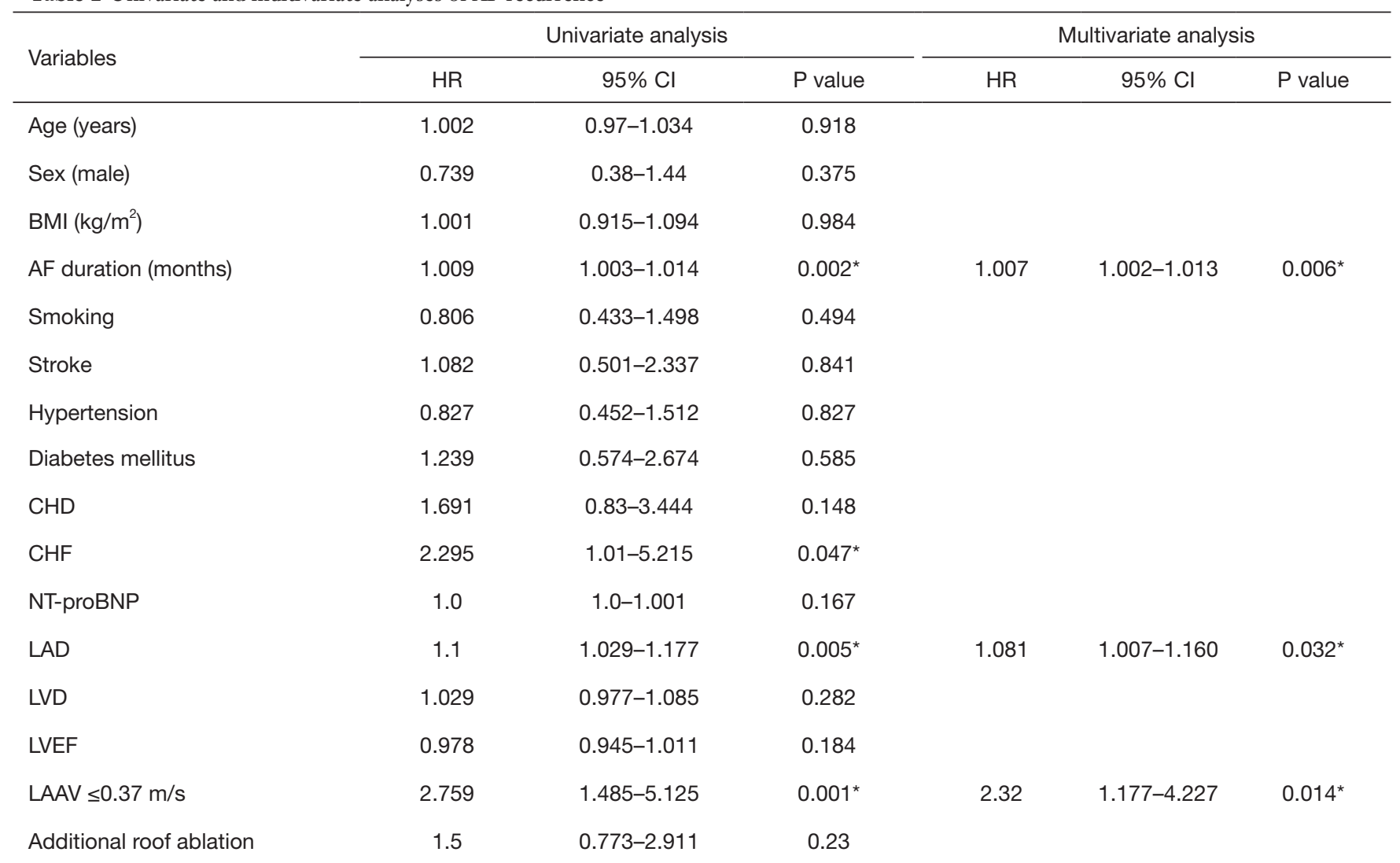

*, $\mathrm{P}<0.05$. AF, atrial fibrillation; HR, hazard ratio; $\mathrm{Cl}$, confidence interval; BMI, body mass index; CHD, coronary heart disease; CHF, chronic heart failure; NT-proBNP, N-terminal pro-brain natriuretic peptide; LAD, left atrial diameter; LVD, left ventricular diameter; LVEF, left ventricular ejection fraction; LAAV, left atrial appendage peak flow velocity. 
Table 3 Relationship between LAAV and other variables

\begin{tabular}{|c|c|c|}
\hline Variables & Correlation coefficient $(r)$ & $P$ value \\
\hline Sex (male) & 0.078 & 0.323 \\
\hline Age (years) & -0.198 & $0.011^{*}$ \\
\hline $\mathrm{BMI}\left(\mathrm{kg} / \mathrm{m}^{2}\right)$ & 0.07 & 0.372 \\
\hline AF duration (months) & -0.081 & 0.303 \\
\hline CHA2DS2-VASc score & -0.25 & $0.001^{\star *}$ \\
\hline HAS-BLED score & -0.185 & $0.017^{\star}$ \\
\hline NT-proBNP & -0.125 & $0.006^{\star *}$ \\
\hline $\mathrm{UA}(\mu \mathrm{mol} / \mathrm{L})$ & 0.005 & 0.947 \\
\hline LVD (mm) & -0.017 & 0.831 \\
\hline LAD (mm) & -0.221 & $0.004^{\star *}$ \\
\hline LVEF (\%) & 0.21 & $0.007^{\star \star}$ \\
\hline \multicolumn{3}{|c|}{$\begin{array}{l}\text { *, P<0.05; }{ }^{*}, \mathrm{P}<0.01 \text {. LAAV, left atrial appendage peak flow } \\
\text { velocity; BMI, body mass index; AF, atrial fibrillation; CHA2DS2- } \\
\text { VASc, Congestive Heart Failure, Hypertension, Age } \geq 75 \text { years } \\
\text { [doubled], Diabetes Mellitus, Stroke [doubled], Vascular Disease, } \\
\text { Age (65-74 years), Sex category [female]; HAS-BLED, elderly, } \\
\text { history of hypertension, history of abnormal renal or liver } \\
\text { function, history of stroke, history of major bleeding, medication } \\
\text { use predisposing to bleeding, alcohol use, Labile international } \\
\text { normalized ratio; NT-proBNP, N-terminal pro-brain natriuretic } \\
\text { peptide; UA, uric acid; LVD, left ventricular diameter; LAD, left } \\
\text { atrial diameter; LVEF, left ventricular ejection fraction. }\end{array}$} \\
\hline
\end{tabular}

months. PeAF individuals with a lower LAAV were at higher risk and more likely to experience AF recurrence.

For patients with PeAF, catheter ablation is the recommended treatment option. However, AF recurrence remains problematic. Identifying the predictors of $\mathrm{AF}$ relapse following radiofrequency ablation is crucial for detecting high-risk patients and can provide therapeutic planning and an increased ablation realization rate. Atrial fibrosis causes structural remodeling, which is a precursor to the onset and persistence of AF. Fibrosis enhances the amount of dysfunctional extracellular matrix, as well as molecular changes, these changes contribute to pathological changes in atrial conduction, which may lead to AF $(32,33)$. According to previous research, patients with LA enlargement are more likely to experience repeated events, and LAD is an important self-regulating indicator of $\mathrm{AF}$ recurrence $(13,34)$. Our findings agree with the findings of these reported researches. Overall, these studies suggest that atrial remodeling is necessary for $\mathrm{AF}$ persistence and recurrence after ablation.
According to previous research $(26,27)$, a reduced LAAV has been linked to a greater risk of AF relapse in PeAF individuals in the 1 year following their initial catheter ablation. Nevertheless, after 1 year of follow-up, it is unknown if LAAV can still predict AF recurrence in individuals having PeAF who had first RFCA. Following a median follow-up of 14 months, prior research indicating LAAV was not an independent predictor of AF relapse following catheter ablation (30). The median follow-up time was 15 months, with a maximum of 24 months. Our findings indicated that LAAV was slightly lower at baseline in the recurrence group than in the non-recurrence group and was revealed to be an independent indicator of $\mathrm{AF}$ recurrence following the initial RFCA in both the univariate and multivariate analyses. The difference in these findings may be due to the differing baseline variables and conditions for patient participation in the two studies. Our research only excluded patients with $\mathrm{AF}$ who had moderate to severe mitral valve disease, while previous studies excluded patients with AF who had any degree of heart valve disease (30); however, we consider our findings more valid since they represent the condition of the vast majority of patients who attend hospital. Secondly, the duration of AF in our sample was longer than in previous research (30). This could be related to extensive atrial structural remodeling because an enhancement in AF's duration has been correlated with a greater extent of LA fibrosis (35). Furthermore, several researches reported that $\mathrm{AF}$ duration is a strong indicator of $\mathrm{AF}$ recurrence $(8,13,36)$. Our multivariate analysis revealed that LAAV persisted as an independent risk factor for AF relapse even following accounting for the AF duration.

LAA is a finger-like projection that arises from the left atrium's main body. LAA compliance can act as a pressure regulator in the left atrium $(20,21)$. During elevated LA pressure and volume overload or atrial fibrosis, the LAA can increase its hemodynamic role by modulating LA pressurevolume relations due to its enhanced distensibility (21). During extreme volume loading in the beating heart, the LAA acts as a reservoir, serving as a barrier to keep the LA pressure from rising too far (20). The emptying of the diastolic LAA in AF was linked to and projected a good LA booster pump function (37). The LAA structure changes that precede LA remodeling, both in terms of operation and morphology, have been shown to predict $\mathrm{AF}$ recurrence $(12,14)$. Based on the hemodynamic activity of the LAA and the findings of our work, we think that the LAA is a much more sensitive criterion for the prediction of AF recurrence than morphological remodeling or LA functional in the LA. 
In our sample, the baseline LAAV was positively linked with LVEF and negatively linked with LAD and NTproBNP. LAA functions are diminished in patients with left ventricular dysfunction who have nonvalvular $\mathrm{AF}$ and a retained or reduced LVEF $(38,39)$. The increased LA filling pressure caused by $\mathrm{LV}$ dysfunction can be transferred to the LAA, leading to the change in LAA function (21). Consequently, LAAV might be related to left ventricular activity to some degree.

Our results have some implications for clinical practice, which we discuss below. Using pre-procedural LAAV, it may be possible to distinguish between individuals at high or low risk of $\mathrm{AF}$ relapse and to predict which individuals are prone to benefit from cardiac catheterization. In addition, it is recommended that further linear and/or electrogram-based ablation operations may be needed to decrease AF relapse following ablation in individuals with a low LAAV to reduce recurrence. Finally, individuals with a low LAAV should be closely monitored in order to identify relapse of $\mathrm{AF}$ as soon as possible.

\section{Study limitations}

There are several limitations to this study. First, this is retrospective research performed at a single site with a minimal sample, leading to selection bias. Second, since our focus was on patients with PeAF, we cannot conclude that our results extend to paroxysmal AF patients. Lastly, the ECG and intermittent Holter testing may have understated the recurrence rates to some extent.

\section{Conclusions}

The current research show that a low LAAV is associated with AF relapse and acts as a predictor of AF relapse following the first RFCA for PeAF. This discovery may help refine therapy strategies and care of individuals with PeAF. More prospective trials are needed to verify these findings in the future. In addition, there may be an association between LAAV and left ventricular activity in individuals with PeAF that requires further study.

\section{Acknowledgments}

It is acknowledged that all authors listed meet the authorship criteria according to the latest guidelines of the International Committee of Medical Journal Editors, and all authors are in agreement with the manuscript.
Funding: This study was supported by the Beijing Natural Science Foundation (Z141100002114050).

\section{Footnote}

Reporting Checklist: The authors have completed the STARD reporting checklist. Available at https://dx.doi. org/10.21037/jtd-21-1363

Data Sharing Statement: Available at https://dx.doi. org/10.21037/jtd-21-1363

Conflicts of Interest: All authors have completed the ICMJE uniform disclosure form (available at https://dx.doi. org/10.21037/jtd-21-1363). The authors have no conflicts of interest to declare.

Ethical Statement: The authors are accountable for all aspects of the work in ensuring that questions related to the accuracy or integrity of any part of the work are appropriately investigated and resolved. All procedures performed in this study involving human participants were in accordance with the Declaration of Helsinki (as revised in 2013). The study was approved by the Human Ethics Review Committee of the Chinese PLA General Hospital and informed consent was taken from all the patients.

Open Access Statement: This is an Open Access article distributed in accordance with the Creative Commons Attribution-NonCommercial-NoDerivs 4.0 International License (CC BY-NC-ND 4.0), which permits the noncommercial replication and distribution of the article with the strict proviso that no changes or edits are made and the original work is properly cited (including links to both the formal publication through the relevant DOI and the license). See: https://creativecommons.org/licenses/by-nc-nd/4.0/.

\section{References}

1. Ruddox V, Sandven I, Munkhaugen J, et al. Atrial fibrillation and the risk for myocardial infarction, all-cause mortality and heart failure: a systematic review and metaanalysis. Eur J Prev Cardiol 2017;24:1555-66.

2. Staerk L, Sherer JA, Ko D, et al. Atrial fibrillation: epidemiology, pathophysiology, and clinical outcomes. Circ Res 2017;120:1501-17.

3. Guo Y, Tian Y, Wang H, et al. Prevalence, incidence, and lifetime risk of atrial fibrillation in China: new 
insights into the global burden of atrial fibrillation. Chest 2015;147:109-19.

4. Santangeli P, Marchlinski FE. Techniques for the provocation, localization, and ablation of non-pulmonary vein triggers for atrial fibrillation. Heart Rhythm 2017;14:1087-96.

5. Nishimura M, Lupercio-Lopez F, Hsu JC. Left atrial appendage electrical isolation as a target in atrial fibrillation. JACC Clin Electrophysiol 2019;5:407-16.

6. Calkins H, Hindricks G, Cappato R, et al. 2017 HRS/ EHRA/ECAS/APHRS/SOLAECE expert consensus statement on catheter and surgical ablation of atrial fibrillation: executive summary. Europace 2018;20:157-208.

7. Murakawa Y, Nogami A, Shoda M, et al. Nationwide survey of catheter ablation for atrial fibrillation: the Japanese Catheter Ablation Registry of Atrial Fibrillation (J-CARAF)--report of 1-year follow-up. Circ J 2014;78:1091-6.

8. Yu HT, Kim IS, Kim TH, et al. Persistent atrial fibrillation over 3 years is associated with higher recurrence after catheter ablation. J Cardiovasc Electrophysiol 2020;31:457-64.

9. Providência R, Adragão P, de Asmundis C, et al. Impact of body mass index on the outcomes of catheter ablation of atrial fibrillation: a European observational multicenter study. J Am Heart Assoc 2019;8:e012253.

10. Pallisgaard JL, Gislason GH, Hansen J, et al. Temporal trends in atrial fibrillation recurrence rates after ablation between 2005 and 2014: a nationwide Danish cohort study. Eur Heart J 2018;39:442-9.

11. Creta A, Providência R, Adragão P, et al. Impact of type2 diabetes mellitus on the outcomes of catheter ablation of atrial fibrillation (european observational multicentre study). Am J Cardiol 2020;125:901-6.

12. Bajraktari G, Bytyçi I, Henein MY. Left atrial structure and function predictors of recurrent fibrillation after catheter ablation: a systematic review and meta-analysis. Clin Physiol Funct Imaging 2020;40:1-13.

13. Mohanty S, Della Rocca DG, Gianni C, et al. Predictors of recurrent atrial fibrillation following catheter ablation. Expert Rev Cardiovasc Ther 2021;19:237-46.

14. Ma XX, Boldt LH, Zhang YL, et al. Clinical relevance of left atrial strain to predict recurrence of atrial fibrillation after catheter ablation: a meta-analysis. Echocardiography 2016;33:724-33.

15. Njoku A, Kannabhiran M, Arora R, et al. Left atrial volume predicts atrial fibrillation recurrence after radiofrequency ablation: a meta-analysis. Europace
2018;20:33-42.

16. Nedios S, Koutalas E, Sommer P, et al. Asymmetrical left atrial remodelling in atrial fibrillation: relation with diastolic dysfunction and long-term ablation outcomes. Europace 2017;19:1463-9.

17. Romero J, Gabr M, Patel K, et al. Efficacy and safety of left atrial appendage electrical isolation during catheter ablation of atrial fibrillation: an updated meta-analysis. Europace 2021;23:226-37.

18. Di Biase L, Burkhardt JD, Mohanty P, et al. Left atrial appendage isolation in patients with longstanding persistent $\mathrm{AF}$ undergoing catheter ablation: BELIEF trial. J Am Coll Cardiol 2016;68:1929-40.

19. Wang YC, Lin LC, Lin MS, et al. Identification of good responders to rhythm control of paroxysmal and persistent atrial fibrillation by transthoracic and transesophageal echocardiography. Cardiology 2005;104:202-9.

20. Hondo T, Okamoto M, Yamane T, et al. The role of the left atrial appendage. A volume loading study in openchest dogs. Jpn Heart J 1995;36:225-34.

21. Davis CA 3rd, Rembert JC, Greenfield JC Jr. Compliance of left atrium with and without left atrium appendage. Am J Physiol 1990;259:H1006-8.

22. Kiedrowicz RM, Wielusinski M, Wojtarowicz A, et al. Left and right atrial appendage functional features as predictors for voltage-defined left atrial remodelling in patients with long-standing persistent atrial fibrillation. Heart Vessels 2021;36:853-62.

23. Hohendanner F, Romero I, Blaschke F, et al. Extent and magnitude of low-voltage areas assessed by ultra-highdensity electroanatomical mapping correlate with left atrial function. Int J Cardiol 2018;272:108-12.

24. Huang D, Li JB, Zghaib T, et al. The extent of left atrial low-voltage areas included in pulmonary vein isolation is associated with freedom from recurrent atrial arrhythmia. Can J Cardiol 2018;34:73-9.

25. Vlachos K, Efremidis M, Letsas KP, et al. Low-voltage areas detected by high-density electroanatomical mapping predict recurrence after ablation for paroxysmal atrial fibrillation. J Cardiovasc Electrophysiol 2017;28:1393-402.

26. Combes S, Jacob S, Combes N, et al. Predicting favourable outcomes in the setting of radiofrequency catheter ablation of long-standing persistent atrial fibrillation: a pilot study assessing the value of left atrial appendage peak flow velocity. Arch Cardiovasc Dis 2013;106:36-43.

27. Kanda T, Masuda M, Sunaga A, et al. Low left atrial appendage flow velocity predicts recurrence of atrial fibrillation after catheter ablation of persistent atrial 
fibrillation. J Cardiol 2015;66:377-81.

28. Ma XX, Zhang YL, Hu B, et al. Association between left atrial appendage emptying velocity, $\mathrm{N}$-terminal plasma brain natriuretic peptide levels, and recurrence of atrial fibrillation after catheter ablation. J Interv Card Electrophysiol 2017;48:343-50.

29. Antonielli E, Pizzuti A, Pálinkás A, et al. Clinical value of left atrial appendage flow for prediction of long-term sinus rhythm maintenance in patients with nonvalvular atrial fibrillation. J Am Coll Cardiol 2002;39:1443-9.

30. Wei Y, Liu S, Yu H, et al. The predictive value of growth differentiation factor-15 in recurrence of atrial fibrillation after catheter ablation. Mediators Inflamm 2020;2020:8360936.

31. Latchamsetty R, Morady F. Catheter ablation of atrial fibrillation. Heart Fail Clin 2016;12:223-33.

32. Mikhailov AV, Kalyanasundaram A, Li N, et al. Comprehensive evaluation of electrophysiological and 3D structural features of human atrial myocardium with insights on atrial fibrillation maintenance mechanisms. J Mol Cell Cardiol 2021;151:56-71.

33. Begg GA, Holden AV, Lip GY, et al. Assessment of atrial fibrosis for the rhythm control of atrial fibrillation. Int J Cardiol 2016;220:155-61.

34. Kim YG, Choi JI, Boo KY, et al. Clinical and echocardiographic risk factors predict late recurrence after radiofrequency catheter ablation of atrial fibrillation. Sci Rep 2019;9:6890.

35. Callegari S, Macchi E, Monaco R, et al. Clinicopathological bird's-eye view of left atrial myocardial fibrosis in 121 patients with persistent atrial fibrillation: developing architecture and main cellular players. Circ Arrhythm Electrophysiol 2020;13:e007588.

36. Tilz RR, Rillig A, Thum AM, et al. Catheter ablation of long-standing persistent atrial fibrillation: 5-year outcomes of the Hamburg Sequential Ablation Strategy. J Am Coll Cardiol 2012;60:1921-9.

37. Donal E, Grimm RA, Yamada H, et al. Usefulness of Doppler assessment of pulmonary vein and left atrial appendage flow following pulmonary vein isolation of chronic atrial fibrillation in predicting recovery of left atrial function. Am J Cardiol 2005;95:941-7.

38. Demirçelik MB, Çetin M, Çiçekcioğlu H, et al. Effect of left ventricular diastolic dysfunction on left atrial appendage function and thrombotic potential in nonvalvular atrial fibrillation. Anadolu Kardiyol Derg 2014;14:256-60.

39. Cemri M, Timurkaynak T, Ozdemir M, et al. Effects of left ventricular systolic dysfunction on left atrial appendage and left atrial functions in patients with chronic nonvalvular atrial fibrillation. Acta Cardiol 2002;57:101-5.

Cite this article as: Yang W, Zhao Q, Yao M, Li X, Zhang Y, Liu C, Shan Z, Wang Y. The prognostic significance of left atrial appendage peak flow velocity in the recurrence of persistent atrial fibrillation following first radiofrequency catheter ablation. J Thorac Dis 2021;13(10):5954-5963. doi: $10.21037 /$ jtd-21-1363 